\title{
AIDS-Related Kaposi Sarcoma T1 (Poor Risk): Gastrointestinal Tract Involvement
}

National Cancer Institute

\section{Source}

National Cancer Institute. AIDS-Related Kaposi Sarcoma T1 (Poor Risk): Gastrointestinal

Tract Involvement. NCI Thesaurus. Code C134975.

AIDS-related Kaposi sarcoma involving the esophagus, stomach, or intestines. 